\title{
Flattening and Implication ${ }^{\star}$
}

\author{
Kouichi Hirata ** \\ Department of Artificial Intelligence, \\ Kyushu Institute of Technology, \\ Kawazu 680-4, Iizuka 820-8502, Japan \\ hirata@ai.kyutech.ac.jp
}

\begin{abstract}
Flattening is a method to make a definite clause functionfree. For a definite clause $C$, flattening replaces every occurrence of a term $f\left(t_{1}, \cdots, t_{n}\right)$ in $C$ with a new variable $v$ and adds an atom $p_{f}\left(t_{1}, \cdots, t_{n}, v\right)$ with the associated predicate symbol $p_{f}$ with $f$ to the body of $C$. Here, we denote the resulting function-free definite clause from $C$ by flat $(C)$. In this paper, we discuss the relationship between flattening and implication. For a definite program $\Pi$ and a definite clause $D$, it is known that if $\operatorname{flat}(\Pi) \models \operatorname{flat}(D)$ then $\Pi \models D$, where $\operatorname{flat}(\Pi)$ is the set of flat $(C)$ for each $C \in \Pi$. First, we show that the converse of the above statement does not hold even if $\Pi=\{C\}$, that is, there exist definite clauses $C$ and $D$ such that $C=D$ but $\operatorname{flat}(C) \not \models$ flat $(D)$. Furthermore, we investigate the conditions of $C$ and $D$ satisfying that $C \models D$ if and only if $\operatorname{flat}(C) \models \operatorname{flat}(D)$. Then, we show that, if (1) $C$ is not self-resolving and $D$ is not tautological, (2) $D$ is not ambivalent, or (3) $C$ is singly recursive, then the statement holds.
\end{abstract}

\section{Introduction}

The purpose of Inductive Logic Programming is to find a hypothesis that explains a given sample. It is a normal setting of Inductive Logic Programming that a hypothesis is a definite clause or a definite program and a sample is the set of (labeled) ground definite clauses. In this setting, the word "explain" is interpreted as either "subsume (denoted by $\succeq$ )" or "imply (denoted by $\models$ )". In the latter case, note that the problem of whether or not a definite clause $C$ implies another definite clause, called an implication problem, is undecidable in general [8]. On the other hand, if $C$ is function-free, then it is obvious that the implication problem is decidable.

Flattening, which has been first introduced in the context of Inductive Logic Programming by Rouveirol [14] (though similar ideas had already been used in other fields), is a method to make a definite clause function-free. For a definite clause $C$, flattening replaces every occurrence of a term $f\left(t_{1}, \cdots, t_{n}\right)$ in $C$

\footnotetext{
* This paper is published in Proc. of 10th International Conference on Algorithmic Learning Theory (ALT'99), Lecture Notes in Artificial Intelligence 1720, 157-168, 1999 (C) Springer-Verlag.

** This work is partially supported by Japan Society for the Promotion of Science, Grants-in-Aid for Encouragement of Young Scientists 11780284.
} 
with a new variable $v$ and adds an atom $p_{f}\left(t_{1}, \cdots, t_{n}, v\right)$ with the associated predicate symbol $p_{f}$ with $f$ to the body of $C$. Additionally, the unit clause $p_{f}\left(x_{1}, \cdots, x_{n}, f\left(x_{1}, \cdots, x_{n}\right)\right) \leftarrow$ is introduced to the background theory for each function symbol $f$ in $C$. We denote the resulting function-free definite clause by flat $(C)$ and the set of unit clauses by $\operatorname{defs}(C)$.

Rouveirol [14] has investigated the several properties of flattening. Muggleton $[9,11]$ has dealt with flattening in order to characterize his inverting implication. De Raedt and Džeroski [2] have analyzed their PAC-learnability of $j k$ clausal theories by transforming possibly infinite Herbrand models into approximately finite models according to flattening. Nienhuys-Cheng and de Wolf [13] have revised the properties of flattening with sophisticated duscussion.

Rouveirol [14] (and Nienhuys-Cheng and de Wolf [13]) has shown that flattening "preserves" subsumption: Let $C$ and $D$ be definite clauses. Then, it holds that:

$$
C \succeq D \text { if and only if } \operatorname{flat}(C) \succeq \operatorname{flat}(D) .
$$

Also Rouveirol [14] (and Nienhuys-Cheng and de Wolf [13]) has claimed that flattening "preserves" implication: Let $\Pi$ be a definite program $\left\{C_{1}, \cdots, C_{n}\right\}$ and $D$ be a definite clause. We denote $\left\{\operatorname{flat}\left(C_{1}\right), \cdots, f l a t\left(C_{n}\right)\right\}$ and $\operatorname{defs}\left(C_{1}\right) \cup$ $\cdots \cup \operatorname{defs}\left(C_{n}\right)$ by flat $(\Pi)$ and $\operatorname{defs}(\Pi)$, respectively. Then, Rouveirol's Theorem is described as follows:

$$
\Pi \models D \text { if and only if } \operatorname{flat}(\Pi) \cup \operatorname{defs}(\Pi) \models \operatorname{flat}(D) .
$$

As the stronger relationship between flattening and implication than Rouveirol's Theorem, Nienhuys-Cheng and de Wolf [13] have shown the following theorem:

$$
\text { If } \operatorname{flat}(\Pi) \models \operatorname{flat}(D) \text {, then } \Pi \models D \text {. }
$$

If the converse of this theorem holds, then the several learning techniques for propositional logic such as $[1,3]$ are directly applied to Inductive Logic Programming. On the other hand, if the converse holds, then the implication problem $\Pi \models D$ is decidable, because $\operatorname{flat}(\Pi)$ and $\operatorname{flat}(D)$ are function-free. However, it contradicts the undecidability of the implication problem $[8,15]$ or the satisfiability problem [5]. In this paper, we show that the converse does not hold even if $\Pi=\{C\}$, that is, there exist definite clauses $C$ and $D$ such that:

$$
C \models D \text { but } \operatorname{flat}(C) \not \operatorname{flat}(D) \text {. }
$$

Furthermore, we investigate the conditions of $C$ and $D$ satisfying that $C=D$ if and only if $f$ lat $(C) \models$ flat $(D)$. Gottlob [4] has introduced the concepts of selfresolving and ambivalent clauses. A definite clause $C$ is self-resolving if $C$ resolves with a copy of $C$, and ambivalent if there exists an atom in the body of $C$ with the predicate symbol same as one of the head of $C$. As the corollary of Gottlob's results [4], we show that, if $C$ is not self-resolving and $D$ is not tautological, or $D$ is not ambivalent, then the statement holds. Furthermore, note that the $C$ in the counterexample stated above is given as a doubly recursive definite clause, 
that is, the body of $C$ contains two atoms that are unifiable with the head of a variant of $C$. Then, we show that, if $C$ is singly recursive, that is, the body of $C$ contains at most one atom that is unifiable with the head of a variant of $C$, then the statement also holds.

\section{Preliminaries}

A literal is an atom or the negation of an atom. A positive literal is an atom and a negative literal is the negation of an atom. A clause is a finite set of literals. A unit clause is a clause containing one positive literal. A definite clause is a clause containing one positive literal. A set of definite clauses are called a definite program. Conventionally, a definite clause is represented as $A \leftarrow A_{1}, \cdots, A_{m}$, where $A$ and $A_{i}(1 \leq i \leq m)$ are atoms.

Let $C$ be a definite clause $A \leftarrow A_{1}, \cdots, A_{m}$. Then, the atom $A$ is called a head of $C$ and denoted by $h e a d(C)$, and the sequence $A_{1}, \cdots, A_{m}$ of atoms is called a body of $C$ and denoted by $\operatorname{bod} y(C)$.

Let $C$ and $D$ be definite clauses. We say that $C$ subsumes $D$, denoted by $C \succeq D$, if there exists a substitution $\theta$ such that $C \theta \subseteq D$, i.e., every literal in $C \theta$ also appears in $D$. Also we say that $C$ implies $D$ or $D$ is a logical consequence of $C$, denoted by $C \models D$, if every model of $C$ is also a model of $D$. $C$ is logically equivalent to $D$, denoted by $C \equiv D$, if $C=D$ and $D \models C$. For definite programs $\Pi$ and $\Sigma, \Pi \succeq D, \Pi \succeq \Sigma, \Pi \models D$ and $\Pi \models \Sigma$ are defined similarly.

Let $C$ and $D$ be two clauses $\left\{L_{1}, \cdots, L_{i}, \cdots, L_{l}\right\}$ and $\left\{M_{1}, \cdots, M_{j}, \cdots, M_{m}\right\}$ which have no variables in common. If the substitution $\theta$ is an mgu for the set $\left\{L_{i}, \neg M_{j}\right\}$, then the clause $\left(\left(C-\left\{L_{i}\right\}\right) \cup\left(D-\left\{\neg M_{j}\right\}\right)\right) \theta$ is called a (binary) resolvent of $C$ and $D$. All of the resolvents of $C$ and $D$ are denoted by $\operatorname{Res}(C, D)$.

Let $\Pi$ be a definite program and $C$ be a definite clause. An SLD-derivation of $C$ from $\Pi$ is a sequence $\left(R_{1}, C_{0}, \theta_{1}\right), \ldots,\left(R_{k}, C_{k-1}, \theta_{k}\right)$ such that $R_{0} \in \Pi$, $R_{k}=C, C_{i-1}$ is a variant of an element of $\Pi, R_{i} \in \operatorname{Res}\left(R_{i-1}, C_{i-1}\right)$, and $\theta_{i}$ is an mgu of the selected literals of $R_{i-1}$ and $C_{i-1}$ for each $1 \leq i \leq k$. If an SLD-derivation of $C$ from $\Pi$ exists, we write $\Pi \vdash C$. In particular, $\{C\} \vdash D$ is denoted by $C \vdash D$.

Theorem 1 (Subsumption Theorem [13]). Let $\Pi$ be a definite program and $D$ be a definite clause. Then, $\Pi \models D$ if and only if there exists a definite clause $E$ such that $\Pi \vdash E$ and $E \succeq D$.

For a definite clause $C$, the lth self-resolving closure of $C$, denoted by $\mathcal{S}^{l}(C)$, is defined inductively as follows:

1. $\mathcal{S}^{0}(C)=\{C\}$,

2. $\mathcal{S}^{l}(C)=\mathcal{S}^{l-1}(C) \cup\left\{R \in \operatorname{Res}(C, D) \mid D \in \mathcal{S}^{l-1}(C)\right\}(l \geq 1)$.

Here, the logically equivalent clauses are regarded as identical. Note that $C \vdash D$ if and only if $D \in \mathcal{S}^{l}(C)$ for some $l \geq 0$. Then: 
Corollary 1 (Implication between Definite Clauses [12]). Let $C$ and $D$ be definite clauses. Then, $C \models D$ if and only if there exists a definite clause $E$ such that $E \in \mathcal{S}^{l}(C)$ and $E \succeq D$ for some $l \geq 0$.

For each $n$-ary function symbol $f$, the associated $(n+1)$-ary predicate symbol $p_{f}$, called a flattened predicate symbol (on $f$ ), is introduced uniquely in the process of flattening. Also we call a definite clause $C$ or a definite program $\Pi$ regular if $C$ or $\Pi$ contains no flattened predicate symbols.

Let $C$ be a definite clause, $t$ be a term appearing in $C$ and $v$ be a variable not appearing in $C$. Then, $\left.C\right|_{t} ^{v}$ denotes the definite clause obtained from $C$ by replacing all occurrences of $t$ in $C$ with $v$.

There exist several variants (but equivalent) of the definition of flattening:

1. Do we introduce an equality theory $[9,14]$ or not $[2,13]$ ?

2. Do we transform a constant symbol to an atom with an unary predicate symbol $[2,14]$ or not $[13]$ ?

As the definition of flattening, we adopt the definition similar as De Raedt and Džeroski [2] that does not introduce an equality theory and does not transform a constant symbol.

Let $C$ be a definite clause. Then, the flattened clause flat $(C)$ of $C$ is defined as follows:

$$
f l a t(C)= \begin{cases}C & \text { if } C \text { is function-free, } \\ \operatorname{flat}\left(C^{\prime}\right) & \text { if } t=f\left(t_{1}, \cdots, t_{n}\right)(n \geq 1) \text { appears in } C,\end{cases}
$$

where $C^{\prime}=\left.C\right|_{t} ^{v} \cup\left\{\neg p_{f}\left(t_{1}, \cdots, t_{n}, v\right)\right\}$ and each $t_{i}(1 \leq i \leq n)$ is a variable or a constant. Also $\operatorname{defs}(C)$ is the set $\left\{p_{f}\left(x_{1}, \cdots, x_{n}, f\left(x_{1}, \cdots, x_{n}\right)\right) \leftarrow\right.$ । $f\left(t_{1}, \cdots, t_{n}\right)$ appears in $\left.C\right\}$ of unit clauses. Furthermore, the number of calls of flat that is necessary to obtain the function-free clause $f l a t(C)$ of $C$ is called a rank of $C$ and denoted by $\operatorname{rank}(C)$.

For a definite program $\Pi=\left\{C_{1}, \cdots, C_{n}\right\}$, we define flat $(\Pi)$ and $\operatorname{defs}(\Pi)$ as follows:

$$
\begin{aligned}
\operatorname{flat}(\Pi) & =\left\{\operatorname{flat}\left(C_{1}\right), \cdots, \operatorname{flat}\left(C_{n}\right)\right\} \\
\operatorname{defs}(\Pi) & =\operatorname{defs}\left(C_{1}\right) \cup \cdots \cup \operatorname{defs}\left(C_{n}\right) .
\end{aligned}
$$

\section{$3 \quad$ Flattening and Implication}

As the relationship between flattening and subsumption, Rouveirol [14] (and Nienhuys-Cheng and de Wolf [13]) has shown the following theorem:

Theorem 2 (Rouveirol [14], Nienhuys-Cheng \& de Wolf [13]). Let $C$ and $D$ be regular definite clauses. Then, $C \succeq D$ if and only if $f$ lat $(C) \succeq f l a t(D)$.

Furthermore, Rouveirol [14] (and Nienhuys-Cheng and de Wolf [13]) has proposed the following relationship between flattening and implication. Let $\Pi$ be a regular definite program and $D$ be a regular definite clause. Then, Rouveirol's Theorem is described as follows: 
Theorem 3 (Rouveirol [14], Nienhuys-Cheng \& de Wolf [13]). Let $\Pi$ be a regular definite program and $D$ be a regular definite clause. Then, $\Pi \models D$ if and only if flat $(\Pi) \cup \operatorname{defs}(\Pi) \models \operatorname{flat}(D)$.

In Appendix, we discuss the proof of Rouveirol's Theorem.

Furthermore, Nienhuys-Cheng and de Wolf [13] have shown the following theorem, which is a stronger relationship between flattening and implication than Rouveirol's Theorem:

Theorem 4 (Nienhuys-Cheng \& de Wolf [13]). Let $\Pi$ be a regular definite program and $D$ be a regular definite clause. If flat $(\Pi) \models$ flat $(D)$, then $\Pi \models D$.

On the other hand, the converse of Theorem 4 does not hold even if $\Pi=\{C\}$ :

Theorem 5. There exist regular definite clauses $C$ and $D$ such that

$$
C \models D \text { but flat }(C) \not \models \operatorname{flat}(D) \text {. }
$$

Proof. Let $C$ and $D$ be the following regular definite clauses:

$$
\begin{aligned}
& C=p\left(f\left(x_{1}\right), f\left(x_{2}\right)\right) \leftarrow p\left(x_{1}, x_{3}\right), p\left(x_{3}, x_{2}\right), \\
& D=p\left(f\left(f\left(x_{1}\right)\right), f\left(f\left(x_{2}\right)\right)\right) \leftarrow p\left(x_{1}, x_{3}\right), p\left(x_{3}, x_{4}\right), p\left(x_{4}, x_{5}\right), p\left(x_{5}, x_{2}\right) .
\end{aligned}
$$

By resolving $C$ to a copy of $C$ itself twice, it holds that $C \vdash D$ as Figure 1 . Hence, it holds that $C \models D$.

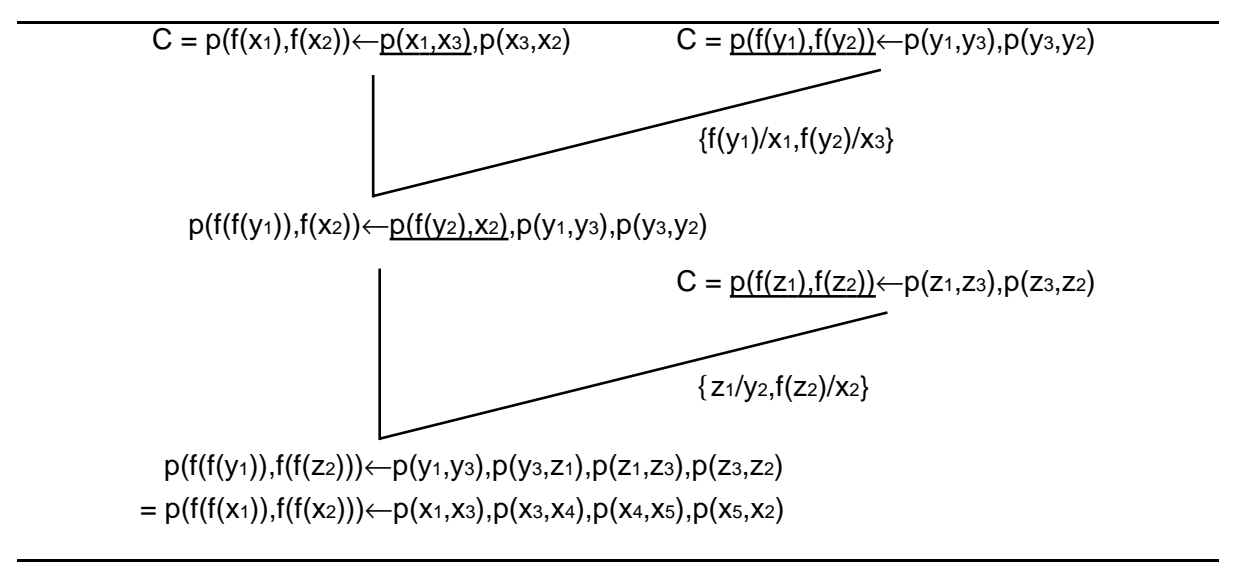

Fig. 1. The SLD-derivation of $D$ from $C$

On the other hand, flat $(C)$ and $f l a t(D)$ are constructed as follows:

$$
\begin{aligned}
\text { flat }(C)=p\left(x_{1}, x_{2}\right) \leftarrow p\left(x_{3}, x_{4}\right), p\left(x_{4}, x_{5}\right), p_{f}\left(x_{3}, x_{1}\right), p_{f}\left(x_{5}, x_{2}\right), \\
\begin{aligned}
\text { flat }(D)=p\left(x_{1}, x_{2}\right) \leftarrow p\left(x_{3}, x_{4}\right), p\left(x_{4}, x_{5}\right), p\left(x_{5}, x_{6}\right), p\left(x_{6}, x_{7}\right), \\
\\
\quad p_{f}\left(x_{3}, x_{8}\right), p_{f}\left(x_{8}, x_{1}\right), p_{f}\left(x_{7}, x_{9}\right), p_{f}\left(x_{9}, x_{2}\right) .
\end{aligned}
\end{aligned}
$$




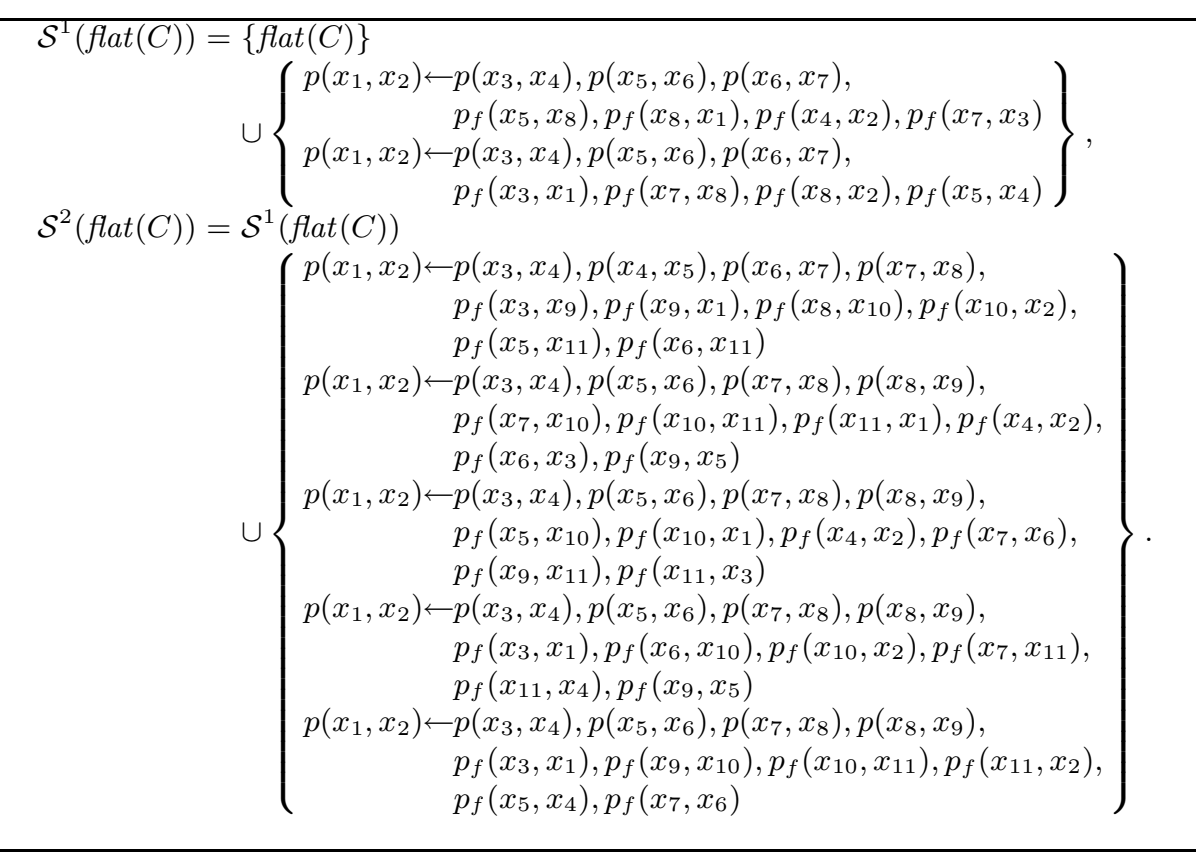

Fig. 2. The first and second self-resolving closures of flat $(C)$

The first and second self-resolving closures of flat $(C)$ are constructed as Figure 2. Then, there exists no definite clause $E \in \mathcal{S}^{2}($ flat $(C))$ such that $E \succeq$ flat $(D)$. By paying our attention to the number of atoms with the predicate $p_{f}$ and its relation, it holds that $f$ lat $(C) \models$ flat $(D)$ if and only if there exists a definite clause $E \in \mathcal{S}^{2}($ flat $(C))$ such that $E \succeq$ flat $(D)$ by Corollary 1 . Hence, we can conclude that there exists no definite clause $E \in \mathcal{S}^{2}($ flat $(C))$ such that $E \succeq$ flat $(D)$, so it holds that $f$ lat $(C) \forall$ flat $(D)$.

Note that, for the definite clauses $C$ and $D$ given in Theorem 5 , it holds that $\{\operatorname{flat}(C)\} \cup \operatorname{defs}(C) \vdash \operatorname{flat}(D)$ as Figure 3, so it holds that $\{\operatorname{flat}(C)\} \cup \operatorname{defs}(C) \models$ flat $(D)$. Hence, $\operatorname{defs}(C)$ is necessary for not only "unflattening" but also unifying with two variables

\section{Improvement}

In this section, we investigate the conditions of definite clauses $C$ and $D$ satisfying that $C \models D$ if and only if $\operatorname{flat}(C)=\operatorname{flat}(D)$.

First, we give the following lemma by Gottlob [4]. A definite clause $C$ is self-resolving if $C$ resolves with a copy of $C$. A definite clause $C$ is ambivalent if there exists an atom in $\operatorname{body}(C)$ with the predicate symbol same as one of $\operatorname{head}(C)$. Then:

Lemma 1 (Gottlob [4]). Let $C$ and $D$ be definite clauses. 


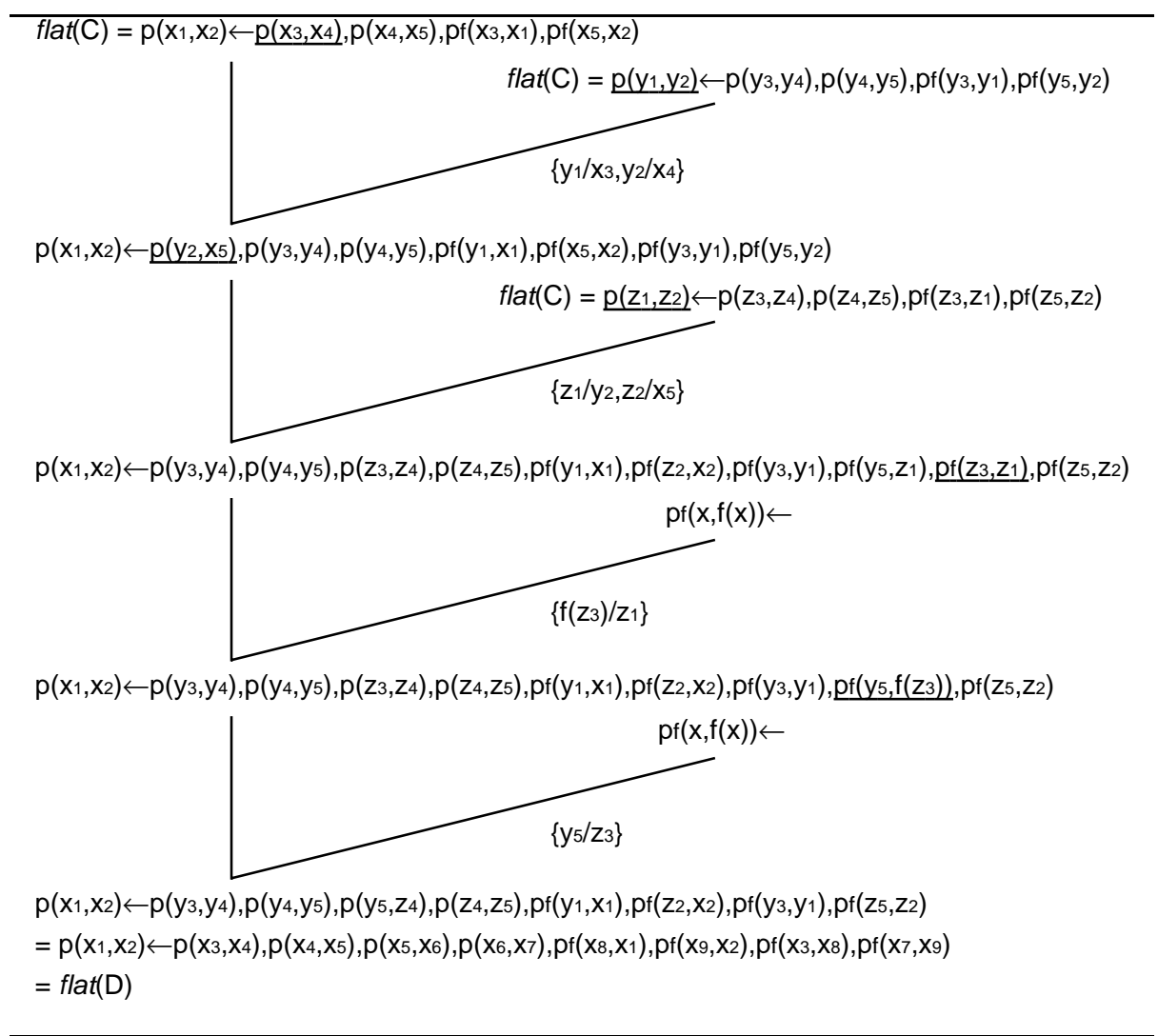

Fig. 3. The SLD-derivation of $\operatorname{flat}(D)$ from $\{\operatorname{flat}(C)\} \cup \operatorname{defs}(C)$ in Theorem 5

1. Suppose that $C$ is not self-resolving and $D$ is not tautological. Then, $C \models D$ if and only if $C \succeq D$.

2. Suppose that $D$ is not ambivalent. Then, $C \models D$ if and only if $C \succeq D$.

By incorporating Lemma 1 with the previous theorems, we obtain the following corollary:

Corollary 2. Let $C$ and $D$ be regular definite clauses.

1. Suppose that $C$ is not self-resolving and $D$ is not tautological. Then, $C \models D$ if and only if flat $(C) \models$ flat $(D)$.

2. Suppose that $D$ is not ambivalent. Then, $C \models D$ if and only if $f$ lat $(C) \models$ flat $(D)$.

Proof. 1. By Lemma 1, $C \models D$ if and only if $C \succeq D$. By Theorem 2, $C \succeq D$ if and only if $\operatorname{flat}(C) \succeq \operatorname{flat}(D)$. By the definition of $\succeq$ and $\models$, if $\operatorname{flat}(C) \succeq \operatorname{flat}(D)$ then $\operatorname{flat}(C) \models \operatorname{flat}(D)$. So it holds that if $C \models D$ then $\operatorname{flat}(C) \models \operatorname{flat}(D)$. Hence, the statement holds by Theorem 4 . 
2. By the definition of ambivalence, the predicate symbol of the head of $D$ is different from all of the predicate symbols appearing in the body of $D$. This condition is preserved in flat $(D)$, because the flattened predicate symbols, which does not appear in $D$, are introduced only in the body of $D$. Hence, flat $(D)$ is not ambivalent. By Lemma 1 and Theorem 2, the statement is obvious.

In Theorem $5, C$ is given as a doubly recursive definite clause, that is, $\operatorname{bod} y(C)$ contains two atoms that are unifiable with head $\left(C^{\prime}\right)$, where $C^{\prime}$ is a variant of $C$. In the remainder of this section, we restrict the form of $C$ to singly recursive. Here, a definite clause $C$ is singly recursive if body $(C)$ contains at most one atom that is unifiable with head $\left(C^{\prime}\right)$, where $C^{\prime}$ is a variant of $C$.

Lemma 2 (Gottlob [4]). If $C \models D$, then head $(C) \succeq \operatorname{head}(D)$ and $\operatorname{bod} y(C) \succeq$ $\operatorname{body}(D)$.

Let $C$ be a singly recursive definite clause. It is obvious that $\left|\mathcal{S}^{l}(C)\right| \leq l+1$ and $\left|\mathcal{S}^{l+1}(C)-\mathcal{S}^{l}(C)\right| \leq 1$ for each $l \geq 0$. Then, the lth self-resolvent $C_{l}$ of $C$ is defined inductively as follows:

1. $C_{0}=C$,

2. $C_{l+1}=\left\{\begin{array}{l}D \text { if } \mathcal{S}^{l+1}(C)-\mathcal{S}^{l}(C)=\{D\}, \\ C \text { otherwise. }\end{array}\right.$

Lemma 3. Let $C$ be a singly recursive regular definite clause with function symbols. Suppose that $C$ contains a term $t=f\left(t_{1}, \cdots, t_{n}\right)$, where each $t_{i}$ is either a variable or a constant. Also let $C^{\prime}$ be a definite clause $\left.C\right|_{t} ^{v} \cup\left\{\neg p_{f}\left(t_{1}, \cdots, t_{n}, v\right)\right\}$. Then, it holds that flat $\left(C_{l}\right) \equiv \operatorname{flat}\left(C_{l}^{\prime}\right)$ for each $l \geq 0$.

Proof. We show the statement by induction on $l$. If $l=0$, then the statement is obvious, since $C_{0}=C, C_{0}^{\prime}=C^{\prime}$ and $\operatorname{flat}(C)=\operatorname{flat}\left(C^{\prime}\right)$.

Suppose that the statement holds for $l \leq k$. It is sufficient to show the case that $C$ is of the form $p(\bar{t}) \leftarrow p(\bar{s})$. Consider $C_{k+1}$ and $C_{k+1}^{\prime}$. By the definition of the $(k+1)$ th self-resolvent, $C_{k+1}$ is a resolvent of $C$ and $C_{k}$, and $C_{k+1}^{\prime}$ is a resolvent of $C^{\prime}$ and $C_{k}^{\prime}$. Then, we can suppose that $C_{k+1}$ is of the form $\left(\operatorname{head}(C) \leftarrow \operatorname{body}\left(C_{k}\right) \mu\right) \theta$, where $\theta$ is an mgu of $h e a d\left(C_{k}\right) \mu$ and $\operatorname{body}(C)$ and $\mu$ is a renaming substitution. Hence, $C_{k+1}^{\prime}$ is of the form (head $\left(C^{\prime}\right) \leftarrow$ $\left.\operatorname{body}\left(C_{k}^{\prime}\right) \mu^{\prime}, p_{f}\left(t_{1}, \cdots, t_{n}, v\right)\right) \theta^{\prime}$, where $\theta^{\prime}$ is a substitution obtained from $\theta$ by replacing the binding $t \mu / x$ in $\theta$ with $v / x$, and $\mu^{\prime}$ is a renaming substitution by adding the binding $u / v$ ( $u$ is a new variable) to $\mu$. By induction hypothesis, it holds that $\operatorname{flat}\left(C_{k}\right) \equiv \operatorname{flat}\left(C_{k}^{\prime}\right)$ and $\operatorname{flat}(C) \equiv \operatorname{flat}\left(C^{\prime}\right)$. By the forms of $C_{k+1}$ and $C_{k+1}^{\prime}$, it holds that $\operatorname{flat}\left(C_{k+1}\right) \equiv \operatorname{flat}\left(C_{k+1}^{\prime}\right)$.

Lemma 4. For a singly recursive definite clause $C$, it holds that flat $\left(C_{l}\right) \equiv$ $(\text { flat }(C))_{l}$ for each $l \geq 0$.

Proof. We show the statement by induction on $\operatorname{rank}(C)$. If $\operatorname{rank}(C)=0$, then the statement is obvious, because flat $\left(C_{l}\right)=C_{l}$ and flat $(C)=C$ for each $l \geq 0$.

Suppose that the statement holds for $C$ such that $\operatorname{rank}(C) \leq k$. Let $C$ be a singly recursive definite clause such that $\operatorname{rank}(C)=k+1$. Since $C$ contains 
some function symbols, suppose that $C$ contains the term $t=f\left(t_{1}, \cdots, t_{n}\right)$, where each $t_{i}$ is either a variable or a constant. Let $C^{\prime}$ be a definite clause $\left.C\right|_{t} ^{v} \cup\left\{\neg p_{f}\left(t_{1}, \cdots, t_{n}, v\right)\right\}$. Then, it holds that $\operatorname{flat}\left(C^{\prime}\right) \equiv \operatorname{flat}(C)$ and $\operatorname{rank}\left(C^{\prime}\right)=$ $k$. By Lemma 3 , it holds that flat $\left(C_{l}^{\prime}\right) \equiv \operatorname{flat}\left(C_{l}\right)$ for each $l \geq 0$. By induction hypothesis, it holds that flat $\left(C_{l}\right) \equiv \operatorname{flat}\left(C_{l}^{\prime}\right) \equiv\left(\operatorname{flat}\left(C^{\prime}\right)\right)_{l} \equiv(\operatorname{flat}(C))_{l}$ for each $l \geq 0$. Hence, the statement holds for $\operatorname{rank}(C)=k+1$.

Lemma 5. For a singly recursive definite clause $C$, it holds that $f$ lat $(C)=$ flat $\left(C_{l}\right)$ for each $l \geq 0$.

Proof. We show the statement by induction on $l$. If $l=0$, then $C_{0}=C$, so the statement is obvious.

Suppose that the statement holds for $l \leq k$. Since $C_{k+1}$ is a resolvent of $C$ and $C_{k}$ and by Lemma 4 , flat $\left(C_{k+1}\right)$ is a resolvent of flat $(C)$ and flat $\left(C_{k}\right)$. By the soundness of SLD-resolution $(c f .[7,13])$, it holds that $\left\{\operatorname{flat}(C)\right.$, flat $\left.\left(C_{k}\right)\right\}=$ flat $\left(C_{k+1}\right)$. By induction hypothesis, it holds that flat $(C) \models$ flat $\left(C_{k}\right)$. Hence, it holds that $\operatorname{flat}(C) \models \operatorname{flat}\left(C_{k+1}\right)$, so the statement holds for $l=k+1$.

Theorem 6. Let $C$ be a singly recursive regular definite clause and $D$ be a regular definite clause. Then, $C \models D$ if and only if $\operatorname{flat}(C)=\operatorname{flat}(D)$.

Proof. By Theorem 4, it is sufficient to show the only-if direction. We show it by induction of $\operatorname{rank}(D)$. If $\operatorname{rank}(D)=0$, that is, $D$ is function-free, then so is $C$ by Lemma 2 . Then, flat $(C)=C$ and $f$ lat $(D)=D$, so the statement is obvious.

Suppose that the statement holds for $D$ such that $\operatorname{rank}(D) \leq k$. Let $D$ be a regular definite clause such that $\operatorname{rank}(D)=k+1$. Since $D$ contains some function symbols, suppose that $D$ contains a term $t=f\left(t_{1}, \cdots, t_{n}\right)$, where each $t_{i}(1 \leq i \leq n)$ is a variable or a constant. Also let $D^{\prime}$ be a definite clause $\left.D\right|_{t} ^{v} \cup\left\{\neg p_{f}\left(t_{1}, \cdots, t_{n}, v\right)\right\}$. Then, $\operatorname{rank}(D)=k$ and $\operatorname{flat}\left(D^{\prime}\right) \equiv \operatorname{flat}(D)$. Suppose that $C \models D$. Then, by Corollary 1 and the definition of the $l$ th self-resolvent, there exists an index $l \geq 0$ such that $C_{l} \succeq D$.

As similar as the proof of Lemma 19.6 in [13], we can construct the definite clause $C^{\prime}$ from $C_{l}$ such that $C^{\prime} \succeq D^{\prime}$ and flat $\left(C_{l}\right) \equiv \operatorname{flat}\left(C^{\prime}\right)$ as follows: Suppose that $C_{l} \theta \subseteq D^{\prime}$. Let $\left\{s_{1}, \cdots, s_{m}\right\}$ be the set of distinct terms occurring in $C_{l}$ such that $s_{i} \theta=t$. If $s_{i}$ is a variable, then replace the binding $t / s_{i}$ with $v / s_{i}$. If $s_{i}$ is of the form $f\left(r_{1}, \cdots, r_{n}\right)$, in which case the $r_{j}$ are variables or constants, then replace all occurrences of $s_{i}$ in $C_{l}$ with a new variable $v_{i}$, add $\neg p_{f}\left(r_{1}, \cdots, r_{n}, v_{i}\right)$ in $C_{l}$, and add the binding $v / v_{i}$ to $\theta$. We call the definite clause resulting from these $m$ adjustments $C^{\prime}$. Finally, replace all occurrences of $t$ in bindings in $\theta$ with $y$, and call the resulting substitution $\theta^{\prime}$. Then, it holds that $C^{\prime} \theta^{\prime} \subseteq D^{\prime}$, so $C^{\prime} \succeq D^{\prime}$. Hence, $C^{\prime} \models D^{\prime}$. Furthermore, by the construction of $C^{\prime}$, it holds that flat $\left(C_{l}\right) \equiv \operatorname{flat}\left(C^{\prime}\right)$.

By induction hypothesis, it holds that flat $\left(C^{\prime}\right)=\operatorname{flat}\left(D^{\prime}\right)$, so flat $\left(C_{l}\right)=$ flat $(D)$. By Lemma 5 , it holds that $f$ lat $(C) \models$ flat $(D)$. Hence, the statement holds for $\operatorname{rank}(D)=k+1$. 


\section{Conclusion}

In this paper, we have investigated the relationship between flattening and implication $[13,14]$. Let $\Pi$ be a regular definite program and $C$ and $D$ be definite clauses. As the stronger relationship between flattening and implication than Rouveirol's Theorem [14], Nienhuys-Cheng and de Wolf [13] have shown the following theorem:

$$
\text { If } \operatorname{flat}(\Pi) \models \operatorname{flat}(D) \text {, then } \Pi \models D \text {. }
$$

In this paper, we have shown that there exist definite clauses $C$ and $D$ such that:

$$
C \models D \text { but } \operatorname{flat}(C) \not \models \operatorname{flat}(D) .
$$

Furthermore, we have shown that if $C$ and $D$ satisfy one of the following conditions, then it holds that $C \models D$ if and only if $\operatorname{flat}(C) \models \operatorname{flat}(D)$ :

1. $C$ is not self-resolving and $D$ is not tautological,

2. $D$ is not ambivalent,

3. $C$ is singly recursive.

Note that the class of definite clauses that flattening preserves implication is corresponding to the class that the implication problem is decidable $[4,6,7]$, and the class of definite clauses that flattening does not preserve implication in the above sense is corresponding to the class that the implication problem is undecidable $[8,15]$. It is a future work to investigate the relationship between the classes of definite clauses that flattening preserves implication and that implication is decidable.

\section{Acknowledgment}

The author would thank to anonymous referees for valuable comments.

\section{References}

1. Angluin, D., Frazier, M. and Pitt, L.: Learning conjunctions of Horn clauses, Machine Learning 9, 147-164, 1992.

2. De Raedt, L. and Džeroski, S.: First-order $j k$-clausal theories are PAC-learnable, Artificial Intelligence 90, 375-392, 1994.

3. Frazier, M. and Pitt, L.: Learning from entailment: An application to propositional Horn sentences, Proc. 10th International Conference on Machine Learning, 120127, 1993

4. Gottlob, G.: Subsumption and implication, Information Processing Letters 24, 109111, 1987.

5. Hanschke, P. and Würtz, J.: Satisfiability of the smallest binary program, Information Processing Letters 45, 237-241, 1993.

6. Leitsch, A.: Implication algorithms for classes of Horn clauses, Statistik, Informatik und Ökonomie, Springer, 172-189, 1988. 
7. Leitsch, A.: The resolution calculus, Springer-Verlag, 1997.

8. Marcinkowski, J. and Pacholski, L.: Undecidability of the Horn-clause implication problem, Proc. 33rd Annual IEEE Symposium on Foundations of Computer Science, 354-362, 1992.

9. Muggleton, S.: Inverting implication, Proc. 2nd International Workshop on Inductive Logic Programming, ICOT Technical Memorandum TM-1182, 1992.

10. Muggleton, S. (ed.): Inductive logic programming, Academic Press, 1992.

11. Muggleton, S.: Inverse entailment and Progol, New Generation Computing 13, 245-286, 1995.

12. Muggleton, S. and Page Jr., C., D.: Self-saturation of definite clauses, Proc. 4th International Workshop on Inductive Logic Programming, 162-174, 1994.

13. Nienhuys-Cheng, S.-H. and de Wolf, R.: Foundations of inductive logic programming, Lecture Notes in Artificial Intelligence 1228, 1997.

14. Rouveirol, C.: Extensions of inversion of resolution applied to theory completion, in [10], 63-92.

15. Schmidt-Schauss, M.: Implication of clauses is undecidable, Theoretical Computer Science 59, 287-296, 1988.

\section{Appendix: Rouveirol's Theorem}

Rouveirol's original proof is insufficient for Rouveirol's Theorem. On the other hand, Nienhuys-Cheng and de Wolf [13] have shown Rouveirol's Theorem as the concequence of Theorem 4 and the following lemma:

Lemma 6. Let $\Pi$ be a regular definite program. Then, $f(a t)(\Pi) \cup \operatorname{defs}(\Pi) \models \Pi$.

On the other hand, we obtain the following theorem:

Theorem 7. There exist regular definite clauses $C$ and $D$ such that

$$
C \vdash D \text { but }\{\text { flat }(C)\} \cup \operatorname{defs}(C) \forall \operatorname{flat}(D) .
$$

Proof. Let $C$ and $D$ be the following regular definite clauses:

$$
\begin{aligned}
& C=p\left(f\left(x_{1}, x_{3}\right), f\left(x_{3}, x_{2}\right)\right) \leftarrow p\left(x_{1}, x_{3}\right), p\left(x_{3}, x_{2}\right), \\
& D=p\left(f\left(f\left(x_{1}, x_{2}\right), f\left(x_{2}, x_{3}\right)\right), f\left(f\left(x_{2}, x_{3}\right), f\left(x_{3}, x_{4}\right)\right)\right) \\
& \leftarrow p\left(x_{1}, x_{2}\right), p\left(x_{2}, x_{3}\right), p\left(x_{2}, x_{3}\right), p\left(x_{3}, x_{4}\right) .
\end{aligned}
$$

By resolving $C$ with $C$ itself twice, we can show that $C \vdash D$.

On the other hand, flat $(C)$ and $f(a t(D)$ are constructed as follows:

$$
\begin{aligned}
\text { flat }(C)=p\left(x_{1}, x_{2}\right) \leftarrow & p\left(x_{3}, x_{4}\right), p\left(x_{4}, x_{5}\right), p_{f}\left(x_{3}, x_{4}, x_{1}\right), p_{f}\left(x_{4}, x_{5}, x_{2}\right), \\
\text { flat }(D)=p\left(x_{1}, x_{2}\right) \leftarrow & p\left(x_{3}, x_{4}\right), p\left(x_{4}, x_{5}\right), p\left(x_{4}, x_{5}\right), p\left(x_{5}, x_{6}\right), \\
& p_{f}\left(x_{3}, x_{4}, x_{7}\right), p_{f}\left(x_{4}, x_{5}, x_{8}\right), p_{f}\left(x_{5}, x_{6}, x_{9}\right), \\
& p_{f}\left(x_{7}, x_{8}, x_{1}\right), p_{f}\left(x_{8}, x_{9}, x_{2}\right) .
\end{aligned}
$$

Also $\operatorname{defs}(C)=\left\{p_{f}(x, y, f(x, y)) \leftarrow\right\}$.

The first and second self-resolving closures of flat $(C)$ are constructed as Figure 4 . By paying our attention to the number of atoms with the predicate $p_{f}$ and its relation, it holds that $\{\operatorname{flat}(C)\} \cup \operatorname{defs}(C) \vdash \operatorname{flat}(D)$ if and only if there exists 


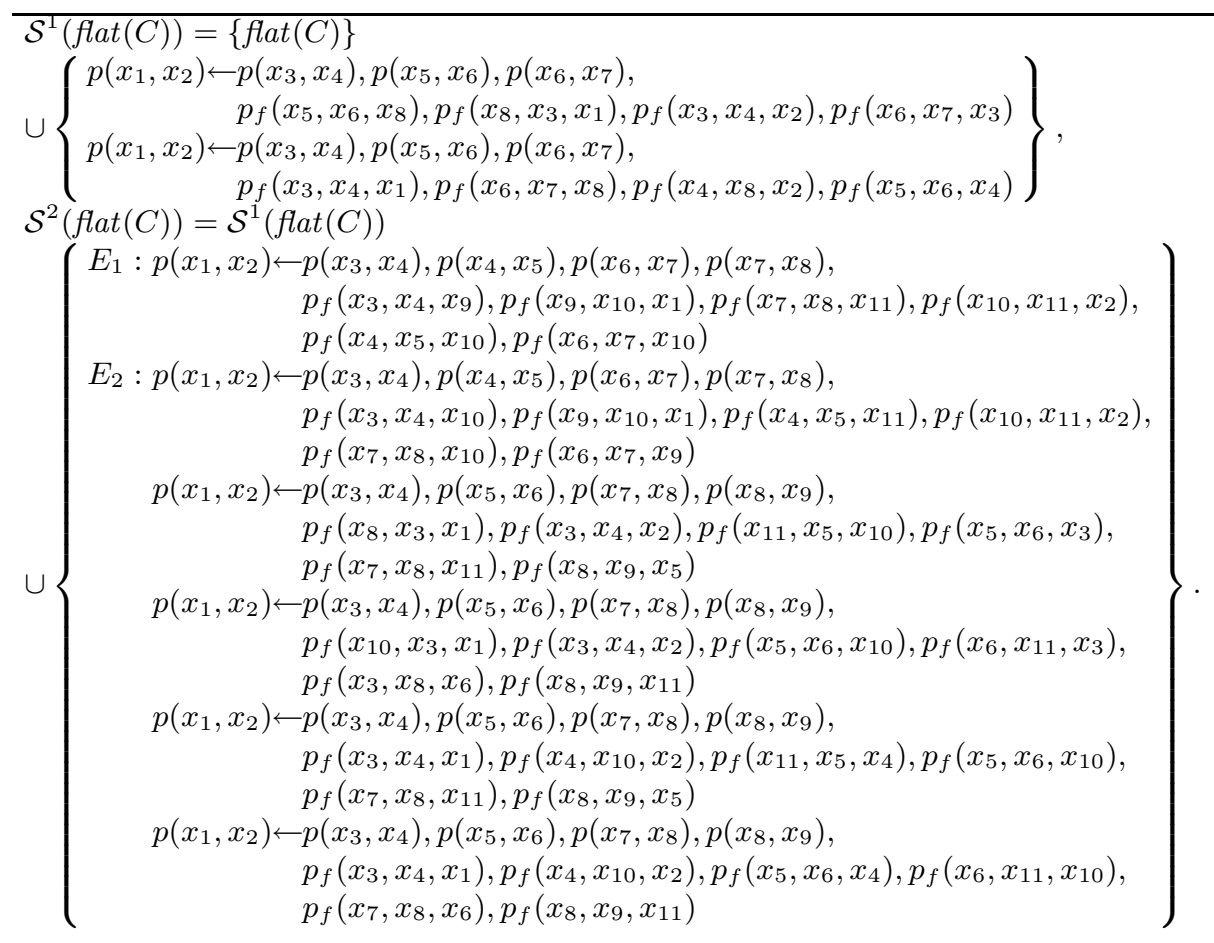

Fig. 4. The first and second self-resolving closures of flat $(C)$

a definite clause $E \in \mathcal{S}^{2}($ flat $(C))$ such that flat $(D)$ is obtained by resolving $E$ with $p_{f}(x, y, f(x, y)) \leftarrow$ some times. Note that we cannot obtain the above $E$ from each element in $\mathcal{S}^{2}($ flat $(C))$ except $E_{1}$ and $E_{2}$. Furthermore, the resolvent of $E_{i}(i=1,2)$ with $p_{f}(x, y, f(x, y)) \leftarrow$ twice, where the selected atoms in $E_{i}$ are atoms of which the third argument's term is $x_{10}$, contains a term with $f$. Hence, it holds that $\{\operatorname{flat}(C)\} \cup \operatorname{defs}(C) \not \operatorname{flat}(D)$.

Hence, we cannot conclude Rouveirol's Theorem from Theorem 4 and Lemma 6 .

Noet that the definite clauses $C$ and $D$ in Theorem 7 are not a counterexample of the if-direction of Rouveirol's Theorem, because $E_{1}$ and $E_{2}$ subsume flat $(D)$ by the following substitutions $\sigma_{1}$ and $\sigma_{2}$ :

$$
\begin{aligned}
& \sigma_{1}=\left\{x_{4} / x_{6}, x_{5} / x_{7}, x_{6} / x_{8}, x_{7} / x_{9}, x_{8} / x_{10}, x_{9} / x_{11}\right\}, \\
& \sigma_{2}=\left\{x_{4} / x_{3}, x_{5} / x_{4}, x_{6} / x_{5}, x_{4} / x_{7}, x_{5} / x_{8}, x_{7} / x_{9}, x_{8} / x_{10}, x_{9} / x_{11}\right\} .
\end{aligned}
$$

